\title{
Multidimensional cell profiling
}

High-throughput profiling of cellular markers by automated microscopy adds a powerful new method for probing cellular function.

In this era of high-throughput methods, quantification of cellular changes caused by external perturbations is generally limited to population-level transcriptional and proteomic profiling techniques. However, cells are much more complex than such methods imply. In a recent issue of Science, Steven Altschuler and colleagues at Harvard describe a method of highthroughput cell profiling that enables a far wider range of phenotypic responses in cells to be quantified and analyzed. Rather than grinding up cells to get population averages, this multidimensional approach allows the investigator to measure changes in individual cells.

This breakthrough was the result of a broad collaboration that combined immunocytochemistry, microscopy, image processing and custom data analysis techniques to create an automated microscopy platform that can quantify changes in tens of millions of individual cells in one study. Cells are labeled with fluorescent antibodies to specific proteins and image processing identifies the nucleus, cytoplasm and other regions. An investigator then defines a set of descriptors to track changes in protein expression and cell morphology. Through the use of some clever data processing, graphical representations of the response of each descriptor to external perturbation are constructed. These color-coded profiles, capable of summarizing almost a billion separate data points, describe the cellular response to any perturbation.

In this initial report the authors examined the individual effects of 13 dilutions of 100 drugs on 93 descriptors in one cell type. It turns out that it is possible to group drugs with different structures but common targets by simply examining the color-coded profiles generated. According to coauthor $\mathrm{Wu}$, "It was surprising to many people that we could identify the broad mechanisms of drug action with one cell type."

Despite the potential of cell profiling for drug testing and discovery, this was not the impetus of the work. Altschuler says, "We are actually interested in how cells organize and this was a very pretty detour on the way to building network models that incorporate space and time." They hope to next pick a single important pathway and use descriptors that will extensively mark it, allowing them to deeply probe the network and show how it is organized on a cellular level.

\section{Daniel Evanko}

\section{RESEARCH PAPERS}

Perlman, Z.E. et al. Multidimensional drug profiling by automated microscopy. Science 306, 1194-1198 (2004).

\section{WHEN CHEMISTRY MEETS BIOLOGY, PROTEASES PROSPER}

Researchers at the Scripps Research Institute and the Université Louis Pasteur have jointly developed highthroughput assays to characterize proteases and their substrates.

Proteases have their enzymatic fingers in a lot of biological pies. Their activity contributes to the post-translational modification of many proteins - so many, in fact, that a high-throughput assay was needed to rapidly screen for protease activities and their substrates. This need prompted Jennifer Harris, a biologist from The Scripps Research Institute, and Nicolas Winssinger, a chemist from the Université Louis Pasteur, to join forces. In two recent papers in Chemistry \& Biology they describe techniques to isolate active proteases from cell lysates and determine their substrate specificities.

Both approaches use fluorescent tetrapeptide libraries bar coded with a peptide nucleic acid (PNA) tag. To isolate active cysteine proteases from a sample, Harris et al. used a peptide library designed to mechanistically inhibit these proteases, and incubated it with a cell lysate. They then separated peptides that covalently bound active proteases from those that did not and hybridized these peptides via their PNA tag to a DNA microarray, thus easily identifying the inhibitors' sequences. By coupling the most potent inhibitor to biotin, they could isolate the active proteases and identify them by mass spectrometry (Harris et al., 2004)

Winssinger et al. modified this approach as a means to identify the substrate specificity of a particular protease. They incubated purified proteases with a library of PNA-encoded fluorescent substrate peptides; upon cleavage of a peptide, its fluorescence intensity increased and could easily be detected after hybridization of the PNA tags to the microarray (Winssinger et al., 2004). Once the 'substrate fingerprint' of a protease has been established, complex mixtures can be screened for the presence of the active protease.

Winssinger says these techniques could also be adapted for other enzymes, such as kinases, or even to receptors and their ligands. Most of all, he emphasizes his interdisciplinary collaboration with Harris as the main strength of their work: "It has been the combination of my chemistry and Jennifer Harris' enzymology background that has made this project work. Without each other we wouldn't have been able to make it work."

\section{Nicole Rusk}

RESEARCH PAPERS

Harris, J. et al. Activity profile of dust mite allergen extract using substrate libraries and functional proteomic microarrays. Chem. Biol. 11, 1361-1372 (2004). 